\section{The Demographics of Non-motor Vehicle Associated Railway Injuries Seen at Trauma Centers in the United States 2007 - 2014}

Christopher A. Schneble ${ }^{1}$, Jodi Raymond ${ }^{2}$, Randall T. Loder ${ }^{3}$

1. Orthopaedic Surgery, Yale University School of Medicine/Yale New Haven Hospital, New Haven, USA 2. Pediatric Surgery, Riley Hospital for Children, Indianapolis, USA 3. Orthopaedic Surgery, Riley Hospital for Children, Indianapolis, USA

Corresponding author: Randall T. Loder, rloder@iupui.edu

\begin{abstract}
Introduction

The majority of railway injury studies are limited by small sample size, restricted to a small geographical distribution, or located outside the United States (US). The aim of our study was to assess the demographic patterns associated with non-motor vehicle railway injuries in the US using a national trauma center database.

Materials and Methods
\end{abstract}

Data from the National Trauma Data Bank data from 2007 - 2014 were used; 3,506 patients were identified. For all statistical analyses, a p-value $<0.05$ was considered significant.

Results

The patients were $81 \%$ male with an average age of $38.6+17.1$ years and an Injury Severity Score (ISS) of $16.8+13.8$. Males compared to females were younger ( 37.7 vs 42.5 years, $p=0.000002$ ), had greater length of stays (12.7 vs 9.8 days, $p=0.000006)$, and higher ISS scores $(17.1$ vs $15.4, p=0.0007)$. The geographic distribution within the US was most common in the South (32.0\%) and least in the Northeast (18.9\%). The racial composition was $67.5 \%$ White, $19.1 \%$ Black, $11.5 \%$ Hispanic/Latino, and $1.9 \%$ others. The most common mechanisms of injury were hitting/colliding with rolling stock (38.6\%), followed by a fall in or from a train $(19.5 \%)$, and collision with an object (13.5\%). The majority of patients were pedestrians or passengers (68.5\%); employees accounted for $12.5 \%$. Although the majority were pedestrian/passengers for all regions, the Midwest had a greater proportion of employees (22.0\%) compared to the other regions ( $7.8 \%$ to $12.2 \%$ ) (p $<10^{-6}$ ), and thus injuries were more commonly work-related $\left(24.6 \%\right.$ vs $\left.6.7 \%-13.7 \%, \mathrm{p}<10^{-6}\right)$. Work-related injuries were less severe (ISS 11.2 vs $17.3-\mathrm{p}<10^{-6}$ ) and more commonly occurred due to a fall (32.8\% vs $\left.17.9 \%, \mathrm{p}<10^{-6}\right)$. Alcohol and/or drug involvement was present in $40.7 \%$ and was less in those with workrelated injuries (2.2\%). Overall mortality was $6.4 \%$ and was less in those having a work-related injury ( $2.0 \mathrm{vs}$ $6.6 \% \mathrm{p}=0.000004)$.

Conclusion

For non-motor vehicle USA railway injuries, the average age was 38.5 years; $80.6 \%$ were male. The injuries were least common in the Northeast and most common in the South. Racial distribution mirrored that of the US population. Alcohol involvement was present in $29 \%$, lower than in previous studies. Mortality was $6.4 \%$, also lower than previously reported.

Categories: Epidemiology/Public Health, Trauma

Keywords: railway, injury, demographics, fatality, alcohol, drugs, injury severity score (iss)

\section{Introduction}

Trains are a common form of transportation in the modern world for both passenger and freight carriage. Regarding passenger traffic, the Worldwide Railway Organization reported that in 2016, 7.342 billion passengers were carried on trains in Europe [1]. Europe accounts for $16 \%$ of worldwide passenger travel, giving a worldwide number of 45.9 billion passengers ( 45.9 billion $=7.342$ billion $/ 0.16$ ). According to the 2016 United States (US) Bureau of Transportation Statistics, there were 39.61 billion passenger-miles for all rail modalities (Amtrak, commuter rail, and transit rail) [2]. The numbers for freight were somewhat different. In 2016, European railways carried 2.209 billion tons of freight [1], or $6 \%$ of worldwide freight, giving a worldwide freight carriage of $\sim 36.8$ billion tons ( 36.8 billion $=2.209$ billion $/ 0.06$ ). Such massive volumes of transportation expose humans to significant potential for injury. When such injuries occur, they are associated with significant morbidity and mortality. In the last 10 years within the US, 119,303 railway injury events occurred, resulting in 87,106 nonfatal injuries and 7,451 deaths [3].

The majority of studies involving railway injuries are limited by small sample size, restricted to a small geographical distribution, and located outside the US [4-7]. Sousa et al. [7], Moore et al. [5], and Cina et al. [8] focused only on train-pedestrian events, while Shapiro et al. [4] and Hedelin et al. [6] reported on multiple mechanisms of injury. Maclean et al. [9] and Mohanty et al. [10] focused on only traumatic amputation patients and patients who perished, respectively. The aim of this investigation was to study the demographic patterns associated with non-motor vehicle collision railway injuries seen at trauma centers in the entire US using a national database. As this was an exploratory demographic study, there were no null hypotheses. 


\section{Materials And Methods}

The data for this study were obtained from the National Trauma Data Bank (NTDB) for the time period 2007 2014 and extracted using SAS version 9.4 (SAS Institute, Cary, NC) and further refined with Excel ${ }^{\circledR} 2013$ (Microsoft ${ }^{\circledR}$, Redmond, WA). There were 3,506 patients identified using the International Classification of Disease (ICD), 9th edition E codes for railway injuries (E800 - E807). These codes exclude those involving motor vehicles. Due to the nature of the NTDB, all patients presented to a hospital. The data collected were age, gender, ethnicity, region/location, length of stay (LOS), length of Intensive Care Unit (ICU) stay, ICD-9 diagnosis codes, ICD-9 procedure codes, complications, relation to work, association with alcohol or drug use, Injury Severity Score (ISS), and disposition from the hospital. Regions within the US were categorized as Northeast, West, South, and Midwest as determined by the US Census Bureau (Figure 1). This study was deemed exempt by the Indiana University Institutional Review Board (IRB00000220|IRB-01|Study $\# 1705380487$ ).

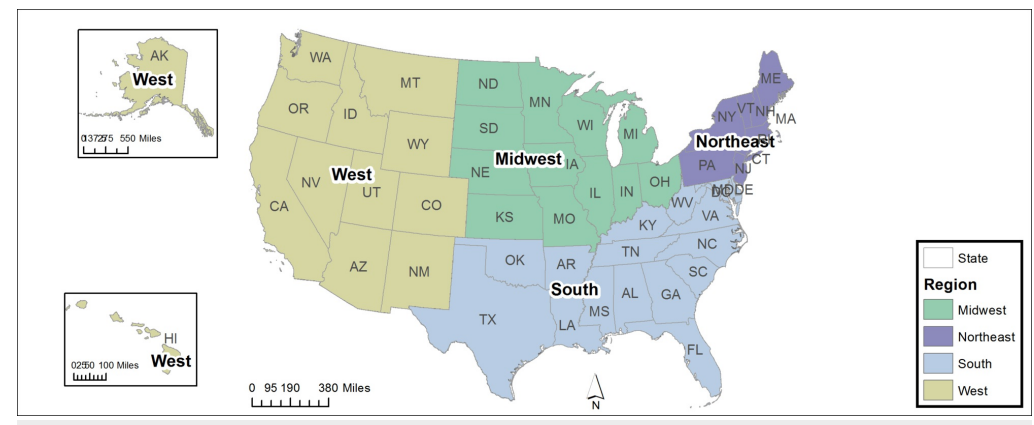

FIGURE 1: The four geographic regions as determined by the United States Census Bureau (http://www.census.gov/geo/reference/webatlas/regions.html)

Figure obtained from the public domain, courtesy of the United States Census Bureau

Continuous data are reported as the mean \pm one standard deviation. Categorical data are reported as frequencies and percentages. Analyses between groups of continuous data were performed using nonparametric tests due to non-normal data distribution (Mann-Whitney U - two groups; Kruskal-Wallis test three or more groups). Differences between groups of discrete data were analyzed by the Fisher's exact test ( $2 \times 2$ tables) and the Pearson's $\chi^{2}$ test (greater than $2 \times 2$ tables). For all statistical analyses, $p<0.05$ was considered statistically significant. Statistical analyses were performed with Systat $10^{\mathrm{rM}}$ (Systat Software, Inc., Chicago, IL).

\section{Results}

\section{Patient demographics}

Most patients were male (80.5\%) with an average age of $38.6 \pm 17.1$ years (range: 0 - 89 years) (Table 1 ). The mean length of stay (LOS) was $12.1 \pm 17.7$ days (range: $1-304$ days). An ICU admission occurred in 1,563 (44.6\%) of the patients with an average stay of $8.6 \pm 11.1$ days (range: 1 - 136 days). The average ISS was 16.8 $\pm 13.8 ; 43.3 \%$ were $<15$ and $56.7 \%$ were $>16$. Racial distribution (when known) was $67.5 \%$ White $19.1 \%$ Black, $11.5 \%$ Hispanic/Latino, $1.8 \%$ Asian, and $0.1 \%$ Polynesian. The injuries occurred most commonly in the South $(32.0 \%)$ and with the fewest in the Northeast (18.9\%). The most common mechanisms of injury were hitting/colliding with rolling stock (38.6\%), followed by a fall in or from a train (19.5\%), and collision with an object (13.5\%). The ICD9 defines 'hit by rolling stock' as being crushed by the train or any part of the train, injured or killed by the train, knocked down by the train, or run over by the train, excluding a pedestrian being hit or by objects set in motion by the train; 'collision with rolling stock' includes a collision between railway trains or railway vehicle, or any derailment of rolling stock colliding with other rolling stock. When known, the majority of patients were pedestrians or passengers (68.5\%); employees accounted for $12.5 \%$. The geographic location where the injury occurred was "other" (47.7\%) (which likely means the railroad itself), followed by the street (22.7\%), industry (11.1\%), and seven other locations comprising the remainder. Alcohol and/or drug involvement was present in 1,428 patients (40.7\%). Alcohol presence was confirmed in 1,001 (28.6\%) and illicit drugs in 661 (18.9\%); 264 (7.5\%) had both alcohol and drug involvement. The injury was fatal in $223(6.4 \%)$; 69 (2.0\%) arrived with no signs of life, while 105 (3.0\%) died in the emergency department (ED). The remaining 49 patients died at some point during their hospital stay prior to discharge. The payor was the government in $40.2 \%$, self-pay in $22.8 \%$, private/commercial insurance in $15.4 \%$, and other payors for the remainder. Differences between various demographic groups are given below, understanding that with such a large data set some of the differences are statistically significant but may not be clinically significant.

\begin{tabular}{|c|c|c|c|c|c|c|c|c|c|c|c|c|c|c|}
\hline Variable & All & Mean/\%* & $\begin{array}{l}\text { Male } \\
\text { (M) }\end{array}$ & $\begin{array}{l}\text { Female } \\
\text { (F) }\end{array}$ & $\begin{array}{l}\% \\
\mathbf{M}\end{array}$ & $\% \mathbf{F}$ & p-value & $\begin{array}{l}\text { White } \\
\text { (W) }\end{array}$ & $\begin{array}{l}\text { Black } \\
\text { (B) }\end{array}$ & $\begin{array}{l}\text { Hispanic/Latino } \\
\text { (H/L) }\end{array}$ & $\begin{array}{l}\% \\
\mathbf{w}\end{array}$ & $\%$ В & $\begin{array}{l}\% \\
H / L\end{array}$ & p-value \\
\hline All & - & - & 2,522 & 610 & 80.5 & 19.5 & - & 2,115 & 599 & 359 & 68.8 & 19.5 & 11.7 & - \\
\hline Age (yrs \pm 1 SD) & 3,491 & $\begin{array}{l}38.6 \pm \\
17.2\end{array}$ & $\begin{array}{l}37.7 \\
\pm \\
16.1\end{array}$ & $\begin{array}{l}42.5 \pm \\
20.7\end{array}$ & - & - & 0.000002 & $\begin{array}{l}39.6 \\
\pm \\
17.4\end{array}$ & $\begin{array}{l}37.4 \\
\pm \\
16.5\end{array}$ & $34.4 \pm 15.3$ & - & - & - & $<10^{-6}$ \\
\hline & & $12.1 \pm$ & 12.7 & $9.8 \pm$ & & & & 111.2 & 13.8 & & & & & \\
\hline
\end{tabular}




\section{Cureus}

\begin{tabular}{|c|c|c|c|c|c|c|c|c|c|c|c|c|c|c|}
\hline LUS (days) & 3,495 & 17.6 & $\begin{array}{l} \pm \\
18.3\end{array}$ & 14.0 & & & u.uov006 & $\begin{array}{l} \pm \\
16.1\end{array}$ & $\begin{array}{l} \pm \\
19.8\end{array}$ & $13 . y \pm 19.3$ & & & & u.us2 \\
\hline ICU LOS (days) & 1,563 & $8.6 \pm 11.1$ & $\begin{array}{l}8.8 \pm \\
11.6\end{array}$ & $\begin{array}{l}7.6 \pm \\
8.6\end{array}$ & & & 0.27 & $\begin{array}{l}8.3 \pm \\
10.2\end{array}$ & $\begin{array}{l}10.1 \\
\pm \\
13.2\end{array}$ & $7.8 \pm 12.6$ & & & & 0.004 \\
\hline ISS & 3,410 & $\begin{array}{l}16.8 \pm \\
13.8\end{array}$ & $\begin{array}{l}17.1 \\
\pm \\
13.9\end{array}$ & $\begin{array}{l}15.4 \pm \\
12.3\end{array}$ & & & 0.0007 & $\begin{array}{l}16.6 \\
\pm \\
13.4\end{array}$ & $\begin{array}{l}17.0 \\
\pm \\
14.8\end{array}$ & $17.0 \pm 14.0$ & & & & 0.69 \\
\hline \multicolumn{15}{|l|}{ ISS Group } \\
\hline$<15$ & 1,449 & 43.3 & 1,199 & 250 & 44.2 & 39.2 & 0.021 & 894 & 233 & 152 & 43.4 & 40.8 & 42.8 & 0.53 \\
\hline$>16$ & 1,899 & 56.7 & 1,511 & 388 & 55.8 & 60.8 & & 1,164 & 338 & 203 & 56.6 & 59.2 & 57.2 & \\
\hline \multicolumn{15}{|l|}{ Gender } \\
\hline Male & 2,522 & 80.5 & - & - & - & - & - & 1,689 & 488 & 309 & 79.9 & 81.5 & 86.1 & 0.020 \\
\hline Female & 610 & 19.5 & - & - & - & - & - & 426 & 111 & 50 & 20.1 & 18.5 & 13.9 & \\
\hline \multicolumn{15}{|l|}{ Race } \\
\hline White & 2,115 & 67.5 & 1,689 & 426 & 67.0 & 69.8 & 0.0001 & - & - & - & - & - & - & \\
\hline Black & 599 & 19.1 & 488 & 111 & 19.3 & 18.2 & & - & - & - & - & - & - & \\
\hline Hispanic/Latino & 359 & 11.5 & 309 & 50 & 12.3 & 8.2 & & - & - & - & - & - & - & \\
\hline Asian & 55 & 1.8 & 33 & 22 & 1.3 & 3.6 & & - & - & - & - & - & - & \\
\hline Polynesian & 4 & 0.1 & 3 & 1 & 0.1 & 0.2 & & - & - & - & - & - & - & \\
\hline \multicolumn{15}{|l|}{ Location in US } \\
\hline Midwest & 643 & 20.5 & 542 & 94 & 21.4 & 16.0 & 0.00002 & 465 & 77 & 37 & 23.8 & 14.8 & 11.0 & $<10^{-6}$ \\
\hline Northeast & 595 & 18.9 & 456 & 136 & 18.0 & 23.1 & & 379 & 83 & 42 & 19.4 & 15.9 & 12.5 & \\
\hline South & 1,004 & 32.0 & 839 & 161 & 33.1 & 27.3 & & 552 & 298 & 109 & 28.2 & 57.1 & 32.3 & \\
\hline West & 896 & 28.6 & 698 & 198 & 27.5 & 33.6 & & 558 & 64 & 149 & 28.6 & 12.3 & 44.2 & \\
\hline \multicolumn{15}{|l|}{ Railway Event } \\
\hline Collision with stock & 339 & 9.8 & 266 & 71 & 9.6 & 10.7 & 0.0015 & 214 & 54 & 29 & 10.1 & 9.0 & 8.1 & 0.001 \\
\hline Collision with object & 466 & 13.5 & 375 & 89 & 13.5 & 13.4 & & 287 & 103 & 36 & 13.6 & 17.2 & 10.0 & \\
\hline Derailment & 65 & 1.9 & 45 & 20 & 1.6 & 3.0 & & 38 & 8 & 6 & 1.8 & 1.3 & 1.7 & \\
\hline Fire/explosion & 14 & 0.4 & 12 & 2 & 0.4 & 0.3 & & 12 & 1 & 1 & 0.6 & 0.2 & 0.3 & \\
\hline Fall & 675 & 19.5 & 524 & 148 & 18.8 & 22.4 & & 455 & 89 & 65 & 21.5 & 14.9 & 18.1 & \\
\hline Hit by stock & 996 & 28.8 & 844 & 147 & 30.4 & 22.2 & & 573 & 193 & 111 & 27.1 & 32.2 & 30.9 & \\
\hline $\begin{array}{l}\text { Other railway } \\
\text { accident }\end{array}$ & 667 & 19.3 & 528 & 138 & 19.0 & 20.8 & & 403 & 105 & 78 & 19.1 & 17.5 & 21.7 & \\
\hline Unspecified & 234 & 6.8 & 186 & 47 & 6.7 & 7.1 & & 133 & 46 & 33 & 6.3 & 7.7 & 9.2 & \\
\hline \multicolumn{15}{|l|}{ Person } \\
\hline Employee & 375 & 12.5 & 344 & 30 & 13.8 & 6.0 & 0.00002 & 281 & 44 & 25 & 15.6 & 8.0 & 7.6 & 0.000001 \\
\hline Pedestrian/Passenger & 2,061 & 68.5 & 1,690 & 360 & 67.6 & 71.4 & & 1,168 & 412 & 231 & 64.7 & 74.6 & 70.4 & \\
\hline Cyclist & 102 & 3.4 & 78 & 35 & 3.1 & 6.9 & & 67 & 18 & 8 & 3.7 & 3.3 & 2.4 & \\
\hline Unknown & 469 & 15.6 & 389 & 79 & 15.6 & 15.7 & & 288 & 78 & 64 & 16.0 & 14.1 & 19.5 & \\
\hline \multicolumn{15}{|l|}{ Work-related } \\
\hline No & 2,714 & 87.1 & 2,110 & 604 & 84.4 & 98.2 & $<10^{-6}$ & 1,621 & 471 & 300 & 84.7 & 89.5 & 91.7 & 0.0002 \\
\hline Yes & 402 & 12.9 & 391 & 11 & 15.6 & 1.8 & & 293 & 55 & 27 & 15.3 & 10.5 & 8.3 & \\
\hline \multicolumn{15}{|l|}{ Injury Location } \\
\hline Home & 74 & 2.2 & 42 & 32 & 1.5 & 4.9 & $<10^{-6}$ & 59 & 6 & 4 & 2.8 & 1.0 & 1.1 & 0.00003 \\
\hline Farm & 7 & 0.2 & 6 & 1 & 0.2 & 0.2 & & 4 & 1 & 1 & 0.2 & 0.2 & 0.3 & \\
\hline Mine & 17 & 0.5 & 17 & 0 & 0.6 & 0.0 & & 13 & 4 & 0 & 0.6 & 0.7 & 0.0 & \\
\hline Industrial & 377 & 11.1 & 354 & 23 & 12.9 & 3.5 & & 267 & 56 & 31 & 12.8 & 9.4 & 8.8 & \\
\hline Recreation & 59 & 1.7 & 44 & 15 & 1.6 & 2.3 & & 50 & 3 & 3 & 2.4 & 0.5 & 0.8 & \\
\hline Street & 769 & 22.7 & 589 & 180 & 21.5 & 27.5 & & 452 & 150 & 93 & 21.7 & 25.3 & 26.3 & \\
\hline
\end{tabular}




\section{Cureus}

\begin{tabular}{|c|c|c|c|c|c|c|c|c|c|c|c|c|c|c|}
\hline Public Building & 288 & 8.5 & 223 & 65 & 8.2 & 9.9 & & 148 & 46 & 32 & 7.1 & 7.8 & 9.1 & \\
\hline Residential & 2 & 0.1 & 2 & 0 & 0.1 & 0.0 & & 2 & 0 & 0 & 0.1 & 0.0 & 0.0 & \\
\hline Other & 1,616 & 47.7 & 1,308 & 308 & 47.8 & 47.1 & & 988 & 299 & 155 & 47.4 & 50.4 & 43.9 & \\
\hline Unknown & 180 & 5.3 & 150 & 30 & 5.5 & 4.6 & & 100 & 28 & 34 & 4.8 & 4.7 & 9.6 & \\
\hline \multicolumn{15}{|l|}{ Alcohol Involvement } \\
\hline No (by test) & 977 & 28.4 & 785 & 192 & 28.2 & 29.0 & $<10^{-6}$ & 604 & 178 & 100 & 28.6 & 27.9 & 28.6 & 0.032 \\
\hline Yes above legal limit & 794 & 23.1 & 694 & 100 & 25.0 & 15.1 & & 479 & 115 & 101 & 22.6 & 28.1 & 22.6 & \\
\hline Yes, trace & 207 & 6.0 & 170 & 37 & 6.1 & 5.6 & & 123 & 36 & 27 & 5.8 & 7.5 & 5.8 & \\
\hline Unknown & 1,464 & 42.5 & 1,131 & 333 & 40.7 & 50.3 & & 909 & 270 & 131 & 43.0 & 36.5 & 43.0 & \\
\hline \multicolumn{15}{|l|}{ Drug Involvement } \\
\hline No by test & 572 & 16.6 & 471 & 101 & 16.9 & 15.3 & 0.071 & 332 & 89 & 68 & 15.7 & 14.9 & 18.9 & 0.51 \\
\hline Yes & 661 & 19.2 & 550 & 111 & 19.8 & 16.8 & & 419 & 124 & 66 & 19.8 & 20.7 & 18.4 & \\
\hline Unknown & 2,209 & 64.2 & 1,759 & 450 & 63.3 & 68.0 & & 1,364 & 386 & 225 & 64.5 & 64.4 & 62.7 & \\
\hline \multicolumn{15}{|l|}{ Disposition } \\
\hline Died & 223 & 6.5 & 187 & 36 & 6.7 & 5.4 & 0.23 & 130 & 45 & 23 & 6.1 & 7.5 & 6.1 & 0.78 \\
\hline Discharged & 2,779 & 80.7 & 2,248 & 531 & 80.9 & 80.2 & & 1,724 & 476 & 291 & 81.5 & 79.5 & 81.5 & \\
\hline $\begin{array}{l}\text { Released from } \\
\text { ED/Unknown }\end{array}$ & 440 & 12.8 & 345 & 95 & 12.4 & 14.4 & & 261 & 78 & 45 & 12.3 & 13.0 & 12.3 & \\
\hline \multicolumn{15}{|l|}{ Payor } \\
\hline Government & 1,384 & 40.2 & 1,127 & 256 & 40.5 & 38.7 & $<10^{-6}$ & 839 & 252 & 139 & 39.7 & 42.1 & 38.7 & $<10^{-6}$ \\
\hline $\begin{array}{l}\text { Blue Cross Blue } \\
\text { Shield }\end{array}$ & 136 & 4.0 & 97 & 39 & 3.5 & 5.9 & & 103 & 14 & 7 & 4.9 & 2.3 & 1.9 & \\
\hline Private/Commercial & 529 & 15.4 & 385 & 144 & 13.8 & 21.8 & & 379 & 71 & 36 & 17.9 & 11.9 & 10.0 & \\
\hline Self & 784 & 22.8 & 676 & 108 & 24.3 & 16.3 & & 443 & 168 & 118 & 20.9 & 28.0 & 32.9 & \\
\hline Other & 230 & 6.7 & 174 & 56 & 6.3 & 8.5 & & 117 & 43 & 24 & 5.5 & 7.2 & 6.7 & \\
\hline Unknown & 380 & 11.0 & 321 & 59 & 11.5 & 8.9 & & 234 & 51 & 35 & 11.1 & 8.5 & 9.7 & \\
\hline
\end{tabular}

TABLE 1: Overall Demographics by Gender and by Race

* average for continuous variables; \% for categorical variables

ED: Emergency Department; ICU: Intensive Care Unit; ISS: Injury Severity Score; LOS: length of stay; SD: standard deviation; US: United States

\section{Analyses by gender and race}

Males compared to females were younger (37.7 vs 42.5 years), had a greater LOS (12.7 vs 9.8 days), and ISS scores (17.1 vs 15.4) (Table 1). Males had a larger proportion of Hispanic/Latinos, injuries occurring in the Midwest and South, injuries that were work-related, hit by the rail stock, and alcohol involvement. Males had a greater percentage of self-pay and a lower percentage of private/commercial insurance. Regarding race, Hispanic/Latinos were the youngest ( $34.4+15.3$ years) and Whites were the oldest $(39.6+17.4$ years) $(\mathrm{p}<$ 10-6). The geographic location of the injury was mostly in the South for Blacks, South, and West for Hispanic/Latinos, and uniform across all four regions for Whites. The patient being an employee was higher in Whites and pedestrian/passengers in Blacks and Hispanic/Latinos. An industrial location was more common in Whites (12.8\%) compared to Hispanic/Latinos (8.8\%). Alcohol involvement was highest in Blacks and lowest in Hispanic/Latinos. Self-pay was most common in Hispanic/Latinos (32.9\%) and lowest in Whites $(20.9 \%)$, with a concomitant increase in private/commercial insurance in Whites (17.9\%) compared to Hispanic/Latinos (10.0\%). There was no difference in ISS, drug involvement, or hospital disposition by race.

\section{Analyses by region of country and relation with work}

Although the majority were pedestrian/passengers for all regions, the Midwest had a greater proportion of employees (22.0\%) compared to the other regions (7.8 to $12.2 \%$ ), and thus injuries were more commonly work-related (24.6\% vs $6.7-13.7 \%$ ) and occurring in industrial locations (17.6\% vs $6.0-11.9 \%$ ) (Table 2). Those that were work-related had less severe injuries (ISS: 11.2 vs 17.3) and more commonly occurred due to a fall (32.8\% vs $17.9 \%)$. Mortality was less in those having a work-related injury ( 2.0 vs $6.6 \%)$. Alcohol involvement was less in those with work-related injuries (2.2\%). 


\section{Cureus}

\begin{tabular}{|c|c|c|c|c|c|c|c|c|c|c|c|c|c|c|}
\hline Age (yrs + 1 SD) & $\begin{array}{l}37.6 \pm \\
16.5\end{array}$ & $\begin{array}{l}39.4 \pm \\
18.5\end{array}$ & $\begin{array}{l}37.5 \pm \\
16.1\end{array}$ & $\begin{array}{l}39.8 \\
\pm \\
17.2\end{array}$ & - & - & - & - & 0.027 & $\begin{array}{l}38.4 \pm \\
17.7\end{array}$ & $\begin{array}{l}41.5 \pm \\
12.6\end{array}$ & - & - & 0.000002 \\
\hline LOS (days) & $\begin{array}{l}11.1 \pm \\
13.7\end{array}$ & $\begin{array}{l}13.0 \pm \\
18.8\end{array}$ & $\begin{array}{l}13.0 \pm \\
16.7\end{array}$ & $\begin{array}{l}11.9 \\
\pm \\
18.7\end{array}$ & - & - & - & - & 0.002 & $\begin{array}{l}12.1 \pm \\
16.9\end{array}$ & $\begin{array}{l}10.2 \pm \\
20.8\end{array}$ & - & - & 0.11 \\
\hline ICU LOS (days) & $\begin{array}{l}8.2 \pm \\
12.6\end{array}$ & $\begin{array}{l}10.1 \pm \\
13.5\end{array}$ & $\begin{array}{l}9.3 \pm \\
11.1\end{array}$ & $\begin{array}{l}7.6 \pm \\
8.9\end{array}$ & - & - & - & - & 0.005 & $\begin{array}{l}8.6 \pm \\
10.6\end{array}$ & $\begin{array}{l}8.4 \pm \\
17.5\end{array}$ & - & - & 0.0055 \\
\hline ISS & $\begin{array}{l}16.1 \pm \\
13.1\end{array}$ & $\begin{array}{l}17.3 \pm \\
14.6\end{array}$ & $\begin{array}{l}16.8 \pm \\
13.3\end{array}$ & $\begin{array}{l}18.0 \\
\pm \\
14.3\end{array}$ & - & - & - & - & 0.045 & $\begin{array}{l}17.3 \pm \\
13.9\end{array}$ & $\begin{array}{l}11.2 \pm \\
9.7\end{array}$ & - & - & $<10^{-6}$ \\
\hline \multicolumn{15}{|l|}{ ISS Group } \\
\hline$<15$ & 265 & 256 & 419 & 418 & 41.5 & 43.5 & 44.4 & 47.1 & 0.16 & 1196 & 94 & 45.1 & 24.0 & $<10^{-6}$ \\
\hline$>16$ & 374 & 333 & 524 & 469 & 58.5 & 56.5 & 55.6 & 52.9 & & 1453 & 298 & 54.9 & 76.0 & \\
\hline \multicolumn{15}{|l|}{ Rallway Event } \\
\hline Collision with Stock & 58 & 59 & 77 & 110 & 9.0 & 9.9 & 7.7 & 12.3 & $<10^{-6}$ & 275 & 36 & 10.1 & 9.0 & $<10^{-6}$ \\
\hline Collision with Object & 82 & 73 & 152 & 122 & 12.8 & 12.3 & 15.1 & 13.6 & & 397 & 31 & 14.6 & 7.7 & \\
\hline Derailment & 11 & 9 & 19 & 7 & 1.7 & 1.5 & 1.9 & 0.8 & & 49 & 13 & 1.8 & 3.2 & \\
\hline Fire/explosion & 6 & 4 & 2 & 1 & 0.9 & 0.7 & 0.2 & 0.1 & & 4 & 8 & 0.1 & 2.0 & \\
\hline Fall & 118 & 139 & 165 & 183 & 18.4 & 23.4 & 16.4 & 20.4 & & 486 & 132 & 17.9 & 32.8 & \\
\hline Hit by Stock & 198 & 166 & 326 & 225 & 30.8 & 27.9 & 32.5 & 25.1 & & 779 & 98 & 28.7 & 24.4 & \\
\hline Other & 125 & 117 & 199 & 176 & 19.4 & 19.7 & 19.8 & 19.6 & & 526 & 73 & 19.4 & 18.2 & \\
\hline Unspecified & 45 & 28 & 64 & 72 & 7.0 & 4.7 & 6.4 & 8.0 & & 201 & 11 & 7.4 & 2.7 & \\
\hline \multicolumn{15}{|l|}{ Person } \\
\hline Employee & 134 & 37 & 113 & 57 & 22.0 & 7.8 & 12.2 & 7.9 & $<10^{-6}$ & 68 & 277 & 2.9 & 71.8 & $<10^{-6}$ \\
\hline Pedestrian/Passenger & 353 & 343 & 691 & 526 & 57.9 & 72.4 & 74.3 & 72.8 & & 1792 & 43 & 77.3 & 11.1 & \\
\hline Cyclist & 20 & 5 & 21 & 44 & 3.3 & 1.1 & 2.3 & 6.1 & & 90 & 0 & 3.9 & 0.0 & \\
\hline Unknown & 103 & 89 & 105 & 96 & 16.9 & 18.8 & 11.3 & 13.3 & & 367 & 66 & 15.8 & 17.1 & \\
\hline \multicolumn{15}{|l|}{ Work-related } \\
\hline No & 454 & 430 & 791 & 776 & 75.4 & 92.5 & 86.3 & 93.3 & $<10^{-6}$ & & & & & \\
\hline Yes & 148 & 35 & 126 & 56 & 24.6 & 7.5 & 13.7 & 6.7 & & & & & & \\
\hline \multicolumn{15}{|l|}{ Injury Location } \\
\hline Home & 21 & 8 & 18 & 11 & 3.3 & 1.4 & 1.8 & 1.3 & $<10^{-6}$ & 71 & 0 & 2.6 & 0.0 & $<10^{-6}$ \\
\hline Farm & 2 & 0 & 1 & 2 & 0.3 & 0.0 & 0.1 & 0.2 & & 5 & 2 & 0.2 & 0.5 & \\
\hline Mine & 2 & 4 & 10 & 0 & 0.3 & 0.7 & 1.0 & 0.0 & & 2 & 13 & 0.1 & 3.3 & \\
\hline Industrial & 112 & 35 & 118 & 80 & 17.6 & 6.0 & 11.9 & 9.2 & & 100 & 249 & 3.7 & 62.9 & \\
\hline Recreation & 12 & 5 & 20 & 14 & 1.9 & 0.9 & 2.0 & 1.6 & & 51 & 1 & 1.9 & 0.3 & \\
\hline Street & 125 & 46 & 236 & 300 & 19.7 & 7.8 & 23.7 & 34.6 & & 691 & 17 & 25.8 & 4.3 & \\
\hline Public Building & 27 & 142 & 30 & 44 & 4.3 & 24.2 & 3.0 & 5.1 & & 265 & 6 & 9.9 & 1.5 & \\
\hline Residential & 0 & 0 & 2 & 0 & 0.0 & 0.0 & 0.2 & 0.0 & & 2 & 0 & 0.1 & 0.0 & \\
\hline Other & 306 & 315 & 495 & 374 & 48.2 & 53.7 & 49.8 & 43.1 & & 1,351 & 93 & 50.4 & 23.5 & \\
\hline Unknown & 28 & 32 & 64 & 43 & 4.4 & 5.5 & 6.4 & 5.0 & & 142 & 15 & 5.3 & 3.8 & \\
\hline \multicolumn{15}{|l|}{ Alcohol Involvement } \\
\hline No (by test) & 197 & 149 & 262 & 281 & 30.6 & 25.0 & 26.1 & 31.4 & 0.00002 & 743 & 168 & 27.3 & 41.8 & $<10^{-6}$ \\
\hline Yes above legal limit & 163 & 135 & 232 & 193 & 25.3 & 22.7 & 23.1 & 21.5 & & 717 & 3 & 26.4 & 0.7 & \\
\hline Yes, trace & 28 & 25 & 61 & 78 & 4.4 & 4.2 & 6.1 & 8.7 & & 187 & 6 & 6.9 & 1.5 & \\
\hline Unknown & 255 & 286 & 449 & 344 & 39.7 & 48.1 & 44.7 & 38.4 & & 1070 & 225 & 39.4 & 56.0 & \\
\hline \multicolumn{15}{|l|}{ Drug Involvement } \\
\hline No by test & 102 & 98 & 162 & 169 & 15.9 & 16.5 & 16.1 & 18.9 & 0.00003 & 461 & 62 & 17.0 & 15.4 & $<10$ \\
\hline
\end{tabular}




\section{Cureus}

\begin{tabular}{|c|c|c|c|c|c|c|c|c|c|c|c|c|c|c|}
\hline Yes & 117 & 78 & 231 & 186 & 18.2 & 13.1 & 23.0 & 20.8 & & 582 & 39 & 21.4 & 9.7 & \\
\hline Unknown & 424 & 419 & 611 & 541 & 65.9 & 70.4 & 60.9 & 60.4 & & 1674 & 301 & 61.6 & 74.9 & \\
\hline \multicolumn{15}{|l|}{ Disposition } \\
\hline Died & 32 & 46 & 65 & 64 & 5.0 & 7.7 & 6.5 & 7.1 & 0.13 & 180 & 8 & 6.6 & 2.0 & 0.000004 \\
\hline Discharged & 531 & 468 & 834 & 713 & 82.6 & 78.7 & 83.1 & 79.6 & & 2160 & 360 & 79.5 & 89.6 & \\
\hline $\begin{array}{l}\text { Released from } \\
\text { ED/Unknown }\end{array}$ & 80 & 81 & 105 & 119 & 12.4 & 13.6 & 10.5 & 13.3 & & 377 & 34 & 13.9 & 8.5 & \\
\hline \multicolumn{15}{|l|}{ Payor } \\
\hline Government & 251 & 244 & 386 & 367 & 39.0 & 41.0 & 38.4 & 41.0 & $<10^{-6}$ & 989 & 286 & 36.4 & 71.1 & $<10^{-6}$ \\
\hline $\begin{array}{l}\text { Blue Cross/Blue } \\
\text { Shield }\end{array}$ & 17 & 27 & 45 & 42 & 2.6 & 4.5 & 4.5 & 4.7 & & 116 & 9 & 4.3 & 2.2 & \\
\hline Private/Commercial & 104 & 123 & 113 & 144 & 16.2 & 20.7 & 11.3 & 16.1 & & 436 & 49 & 16.0 & 12.2 & \\
\hline Self & 135 & 89 & 325 & 164 & 21.0 & 15.0 & 32.4 & 18.3 & & 703 & 6 & 25.9 & 1.5 & \\
\hline Other & 19 & 35 & 72 & 64 & 3.0 & 5.9 & 7.2 & 7.1 & & 200 & 11 & 7.4 & 2.7 & \\
\hline
\end{tabular}

\section{TABLE 2: Demographics by Geographic Region and Work Relationship}

ED: Emergency Department; ICU: Intensive Care Unit; ISS: Injury Severity Score; LOS: length of stay; NWR: not work-related; SD: standard deviation; WR: work-related

Analyses by the mechanism of injury and local geographic location

Those who were injured in a fall were older (43.8 years) than the other major mechanisms of injury (collision with the stock, collision with an object, hit by the stock, or other) (36.6 - 39.6 years) (Table 3). The LOS was highest for those who were hit by the stock (13.8 days vs 8.3 - 13.1); however, when an ICU stay occurred, those who collided with the stock had the highest LOS in the ICU (10.4 days vs $7.0-9.1)$. Those who had a collision with an object or were hit by the stock had more severe injuries (ISS: 19.1 and 18.8 vs 11.5 - 17.5). Alcohol involvement was more common in those who were hit by the stock (52.8\%) than other mechanisms of injury. Hospital mortality was highest in those who collided with an object (8.6\%) or were hit by the stock (8.2\%).

\begin{tabular}{|c|c|c|c|c|c|c|c|c|c|c|c|c|c|c|c|c|c|c|c|}
\hline Variable & $\begin{array}{l}\text { Collision } \\
\text { with } \\
\text { Stock }\end{array}$ & $\begin{array}{l}\text { Collision } \\
\text { with } \\
\text { Object }\end{array}$ & Fall & $\begin{array}{l}\text { Hit by } \\
\text { Stock }\end{array}$ & Other & $\begin{array}{l}\% \\
\text { CS }\end{array}$ & $\%$ co & $\%$ Fall & \%Hit & \%Oth & p-value & Industrial & Street & $\begin{array}{l}\text { Pub } \\
\text { Build }\end{array}$ & Other & \%Ind & $\%$ Str & \%PB & $\%$ \\
\hline Age (yrs \pm 1 SD) & $\begin{array}{l}39.6 \pm \\
16.9\end{array}$ & $\begin{array}{l}37.1 \pm \\
16.4\end{array}$ & $\begin{array}{l}43.8 \\
\pm \\
19.6\end{array}$ & $\begin{array}{l}36.6 \\
\pm \\
15.5\end{array}$ & $\begin{array}{l}38.0 \pm \\
16.5\end{array}$ & & & & & & $<10^{-6}$ & $\begin{array}{l}39.0 \pm \\
14.0\end{array}$ & $\begin{array}{l}39.4 \pm \\
17.2\end{array}$ & $\begin{array}{l}43.1 \\
\pm \\
18.2\end{array}$ & $\begin{array}{l}37.6 \\
\pm \\
16.9\end{array}$ & & & & \\
\hline LOS (days) & $\begin{array}{l}11.9 \pm \\
16.7\end{array}$ & $\begin{array}{l}13.1 \pm \\
18.4\end{array}$ & $\begin{array}{l}8.3 \\
\pm \\
12.3\end{array}$ & $\begin{array}{l}13.8 \\
\pm \\
17.3\end{array}$ & $\begin{array}{l}12.9 \pm \\
18.9\end{array}$ & & & & & & $<10^{-6}$ & $\begin{array}{l}11.0 \pm \\
20.7\end{array}$ & $\begin{array}{l}11.9 \pm \\
17.7\end{array}$ & $\begin{array}{l}12.3 \\
\pm \\
18.1\end{array}$ & $\begin{array}{l}12.8 \\
\pm \\
16.8\end{array}$ & & & & \\
\hline ICU LOS (days) & $\begin{array}{l}10.4 \pm \\
14.0\end{array}$ & $\begin{array}{l}9.1 \pm \\
11.2\end{array}$ & $\begin{array}{l}7.0 \\
\pm \\
9.8\end{array}$ & $\begin{array}{l}8.0 \pm \\
9.8\end{array}$ & $\begin{array}{l}8.5 \pm \\
10.9\end{array}$ & & & & & & 0.0007 & $7.6 \pm 13.6$ & $\begin{array}{l}8.8 \pm \\
10.9\end{array}$ & $\begin{array}{l}8.5 \pm \\
9.6\end{array}$ & $\begin{array}{l}8.6 \pm \\
10.6\end{array}$ & & & & \\
\hline ISS & $\begin{array}{l}16.6 \pm \\
13.2\end{array}$ & $\begin{array}{l}19.1 \pm \\
13.8\end{array}$ & $\begin{array}{l}11.5 \\
\pm \\
9.9\end{array}$ & $\begin{array}{l}18.8 \\
\pm \\
13.9\end{array}$ & $\begin{array}{l}17.5 \pm \\
15.3\end{array}$ & & & & & & $<10^{-6}$ & $\begin{array}{l}12.9 \pm \\
11.4\end{array}$ & $\begin{array}{l}18.6 \pm \\
14.3\end{array}$ & $\begin{array}{l}15.5 \\
\pm \\
13.0\end{array}$ & $\begin{array}{l}18.0 \\
\pm \\
14.4\end{array}$ & & & & \\
\hline \multicolumn{20}{|l|}{ ISS Group } \\
\hline$<15$ & 140 & 233 & 165 & 489 & 287 & 43.2 & 51.8 & 24.7 & 50.6 & 44.2 & $<10^{-6}$ & 112 & 366 & 114 & 743 & 30.5 & 48.7 & 39.7 & 4 \\
\hline$>16$ & 184 & 217 & 503 & 478 & 363 & 56.8 & 48.2 & 75.3 & 49.4 & 55.8 & & 255 & 386 & 173 & 832 & 69.5 & 51.3 & 60.3 & 5 \\
\hline \multicolumn{20}{|l|}{ Railway Accident } \\
\hline Collision with Stock & - & - & - & - & - & - & - & - & - & - & & 32 & 71 & 15 & 150 & 8.5 & 9.2 & 5.2 & 9 \\
\hline $\begin{array}{l}\text { Collision with } \\
\text { Object }\end{array}$ & - & - & - & - & - & - & - & - & - & - & & 22 & 156 & 34 & 216 & 5.8 & 20.3 & 11.8 & 1 \\
\hline Derailment & - & - & - & - & - & - & - & - & - & - & & 6 & 15 & 3 & 21 & 1.6 & 2.0 & 1.0 & 1 \\
\hline Fire/Explosion & - & - & - & - & - & - & - & - & - & - & & 4 & 2 & 1 & 2 & 1.1 & 0.3 & 0.3 & 0 \\
\hline Fall & - & - & - & - & - & - & - & - & - & - & & 127 & 77 & 91 & 304 & 33.7 & 10.0 & 31.6 & 1 \\
\hline Hit by Stock & - & - & - & - & - & - & - & - & - & - & & 104 & 206 & 84 & 513 & 27.6 & 26.8 & 29.2 & 3 \\
\hline
\end{tabular}




\section{Cureus}

\begin{tabular}{|c|c|c|c|c|c|c|c|c|c|c|c|c|c|c|c|c|c|c|c|}
\hline $\begin{array}{l}\text { Other Railway } \\
\text { Accident }\end{array}$ & - & - & - & - & - & - & - & - & - & - & & 68 & 167 & 47 & 316 & 18.0 & 21.7 & 16.3 & 1 \\
\hline Unspecified & - & - & - & - & - & - & - & - & - & - & & 14 & 75 & 13 & 100 & 3.7 & 9.8 & 4.5 & 6 \\
\hline \multicolumn{20}{|l|}{ Injury Location } \\
\hline Home & 32 & 5 & 2 & 15 & 11 & 9.6 & 1.1 & 0.3 & 1.5 & 1.7 & $<10^{-6}$ & - & - & - & - & - & - & - & - \\
\hline Farm & 1 & 1 & 0 & 1 & 4 & 0.3 & 0.2 & 0.0 & 0.1 & 0.6 & & - & - & - & - & - & - & - & - \\
\hline Mine & 2 & 4 & 3 & 0 & 6 & 0.6 & 0.9 & 0.5 & 0.0 & 0.9 & & - & - & - & - & - & - & - & - \\
\hline Industrial & 32 & 22 & 127 & 104 & 68 & 9.6 & 4.8 & 19.3 & 10.6 & 10.3 & & - & - & - & - & - & - & - & - \\
\hline Recreation & 8 & 3 & 10 & 10 & 5 & 2.4 & 0.7 & 1.5 & 1.0 & 0.8 & & - & - & - & - & - & - & - & - \\
\hline Street & 71 & 156 & 77 & 206 & 167 & 21.3 & 33.9 & 11.7 & 21.0 & 25.4 & & - & - & - & - & - & - & - & - \\
\hline Public Building & 15 & 34 & 91 & 84 & 47 & 4.5 & 7.4 & 13.8 & 8.6 & 7.1 & & - & - & - & - & - & - & - & - \\
\hline Residential & 1 & 1 & 0 & 0 & 0 & 0.3 & 0.2 & 0.0 & 0.0 & 0.0 & & - & - & - & - & - & - & - & - \\
\hline Other & 150 & 216 & 304 & 513 & 316 & 45.0 & 47.0 & 46.2 & 52.3 & 48.0 & & - & - & - & - & - & - & - & - \\
\hline Unknown & 21 & 18 & 44 & 47 & 34 & 6.3 & 3.9 & 6.7 & 4.8 & 5.2 & & - & - & - & - & - & - & - & - \\
\hline \multicolumn{20}{|l|}{$\begin{array}{l}\text { Alcohol } \\
\text { Involvement }\end{array}$} \\
\hline No (by test) & 83 & 149 & 200 & 271 & 183 & 24.5 & 24.4 & 20.2 & 21.4 & 19.8 & $<10^{-6}$ & 143 & 229 & 66 & 447 & 37.9 & 22.0 & 29.8 & 2 \\
\hline $\begin{array}{l}\text { Yes, above legal } \\
\text { limit }\end{array}$ & 63 & 103 & 105 & 318 & 142 & 18.6 & 16.9 & 10.6 & 25.1 & 15.4 & & 28 & 198 & 81 & 421 & 7.4 & 11.9 & 25.7 & 2 \\
\hline Yes, trace & 18 & 175 & 342 & 338 & 299 & 5.3 & 28.6 & 34.6 & 26.7 & 32.4 & & 11 & 51 & 21 & 100 & 2.9 & 5.1 & 6.6 & 6 \\
\hline Unknown & 175 & 184 & 342 & 338 & 299 & 51.6 & 30.1 & 34.6 & 26.7 & 32.4 & & 195 & 291 & 120 & 654 & 51.7 & 61.0 & 37.8 & 4 \\
\hline \multicolumn{20}{|l|}{ Drug Involvement } \\
\hline No by test & 44 & 86 & 90 & 191 & 116 & 13.0 & 18.5 & 13.3 & 19.2 & 20.1 & 0.00003 & 59 & 131 & 51 & 282 & 15.6 & 11.9 & 17.0 & 1 \\
\hline Yes & 61 & 110 & 108 & 208 & 117 & 18.0 & 23.6 & 16.0 & 20.9 & 20.3 & & 41 & 162 & 41 & 346 & 10.9 & 10.2 & 21.1 & 2 \\
\hline Unknown & 234 & 270 & 477 & 597 & 343 & 69.0 & 57.9 & 70.7 & 59.9 & 59.5 & & 277 & 476 & 196 & 994 & 73.5 & 78.0 & 61.9 & 6 \\
\hline \multicolumn{20}{|l|}{ Disposition } \\
\hline Died & 15 & 40 & 25 & 82 & 37 & 4.4 & 8.6 & 3.7 & 8.2 & 5.5 & 0.0032 & 17 & 62 & 21 & 110 & 4.5 & 8.1 & 7.3 & 6 \\
\hline Discharged & 275 & 363 & 568 & 795 & 538 & 81.1 & 77.9 & 84.1 & 79.8 & 80.7 & & 330 & 583 & 237 & 1301 & 87.5 & 75.8 & 82.3 & 8 \\
\hline $\begin{array}{l}\text { Released from } \\
\text { ED/Unknown }\end{array}$ & 49 & 63 & 82 & 119 & 92 & 14.5 & 13.5 & 12.1 & 11.9 & 13.8 & & 30 & 124 & 30 & 211 & 8.0 & 16.1 & 10.4 & 1 \\
\hline \multicolumn{20}{|l|}{ Payor } \\
\hline Government & 141 & 161 & 300 & 411 & 259 & 41.6 & 34.5 & 44.4 & 41.3 & 38.8 & 0.0001 & 226 & 259 & 110 & 632 & 59.9 & 40.7 & 33.7 & 3 \\
\hline $\begin{array}{l}\text { Blue Cross/Blue } \\
\text { Shield }\end{array}$ & 25 & 20 & 28 & 30 & 19 & 7.4 & 4.3 & 4.1 & 3.0 & 2.8 & & 9 & 26 & 16 & 66 & 2.4 & 11.9 & 3.4 & 4 \\
\hline Private/Commercial & 61 & 91 & 100 & 135 & 101 & 18.0 & 19.5 & 14.8 & 13.6 & 15.1 & & 58 & 105 & 63 & 237 & 15.4 & 18.6 & 13.7 & 1 \\
\hline Self & 61 & 123 & 127 & 248 & 157 & 18.0 & 26.4 & 18.8 & 24.9 & 23.5 & & 37 & 204 & 48 & 420 & 9.8 & 13.6 & 26.5 & 2 \\
\hline Other & 18 & 32 & 39 & 50 & 58 & 5.3 & 6.9 & 5.8 & 5.0 & 8.7 & & 8 & 79 & 22 & 105 & 2.1 & 5.1 & 10.3 & 6 \\
\hline Unknown & 33 & 39 & 81 & 122 & 73 & 9.7 & 8.4 & 12.0 & 12.2 & 10.9 & & 39 & 96 & 29 & 162 & 10.3 & 10.2 & 12.5 & 1 \\
\hline
\end{tabular}

TABLE 3: Demographics by Mechanism of Injury and Local Geographic Location

CO: collision with object; CS: collision with stock; ICU: Intensive Care Unit; Ind: industrial; ISS: Injury Severity Score; LOS: length of stay: Oth: other; PB: public building; SD: standard deviation; Str: street

\section{Mortality}

The overall mortality was $6.4 \%$ (223 of 3,506). Significant differences between those who survived and those who died are shown in Table 4. Those who died had lower LOS and ICU LOS but a higher ISS. Those who were hit by the stock or collided with an object had higher mortality, as did pedestrian/passengers and cyclists, while employees and those with work-related injuries had a lower mortality rate. Those who were self-pay (likely uninsured) had a higher mortality rate, while those insured by government programs, Blue Cross/Blue Shield (BCBS), and private/commercial payors had a lower mortality rate. 


\section{Cureus}

\begin{tabular}{|c|c|c|c|c|c|}
\hline Variable & Survived & Died & $\%$ Survived & $\%$ Died & $\mathrm{p}$-value \\
\hline Age (yrs \pm 1 SD) & $38.6 \pm 17.2$ & $40.5 \pm 17.5$ & - & - & 0.13 \\
\hline LOS (days) & $14.0 \pm 17.7$ & $4.7 \pm 7$ & - & - & $<10^{-6}$ \\
\hline ICU LOS (days) & $8.9 \pm 11.5$ & $5.7 \pm 8.0$ & - & - & $<10^{-6}$ \\
\hline ISS & $15.2 \pm 11.5$ & $38.2 \pm 16.0$ & - & - & $<10^{-6}$ \\
\hline \multicolumn{6}{|l|}{ ISS Group } \\
\hline$<15$ & 1,631 & 13 & 60.0 & 6.1 & $<10^{-6}$ \\
\hline$>16$ & 1,088 & 199 & 40.0 & 93.9 & \\
\hline Gender & $\mathrm{n}$ & $\mathrm{n}$ & & & \\
\hline Male & 2,248 & 187 & 80.9 & 83.9 & 0.33 \\
\hline Female & 531 & 36 & 19.1 & 16.1 & \\
\hline \multicolumn{6}{|l|}{ Race } \\
\hline White & 1,724 & 130 & 68.0 & 64.4 & 0.76 \\
\hline Black & 476 & 45 & 18.8 & 22.3 & \\
\hline Hispanic/Latino & 291 & 23 & 11.5 & 11.4 & \\
\hline Asian & 43 & 4 & 1.7 & 2.0 & \\
\hline Polynesian & 3 & 0 & 0.1 & 0.0 & \\
\hline \multicolumn{6}{|l|}{ Location in the US } \\
\hline Midwest & 531 & 32 & 20.9 & 15.5 & 0.18 \\
\hline Northeast & 468 & 46 & 18.4 & 22.2 & \\
\hline South & 834 & 65 & 32.8 & 31.4 & \\
\hline West & 713 & 64 & 28.0 & 30.9 & \\
\hline \multicolumn{6}{|l|}{ Railway Accident } \\
\hline Collision with Stock & 275 & 15 & 9.9 & 6.7 & 0.00004 \\
\hline Collision with Object & 363 & 40 & 13.1 & 17.9 & \\
\hline Derailment & 60 & 0 & 2.2 & 0.0 & \\
\hline Fire/Explosion & 8 & 0 & 0.3 & 0.0 & \\
\hline Fall & 568 & 25 & 20.4 & 11.2 & \\
\hline Hit by Stock & 795 & 82 & 28.6 & 36.8 & \\
\hline Other Railway Accident & 538 & 37 & 19.4 & 16.6 & \\
\hline Unspecified & 173 & 24 & 6.2 & 10.8 & \\
\hline \multicolumn{6}{|l|}{ Person } \\
\hline Employee & 332 & 6 & 13.0 & 2.9 & $<10^{-6}$ \\
\hline Pedestrian/Passenger & 1,607 & 174 & 63.1 & 84.1 & \\
\hline Cyclist & 72 & 12 & 2.8 & 5.8 & \\
\hline Unknown & 388 & 19 & 15.2 & 9.2 & \\
\hline \multicolumn{6}{|l|}{ Work-related } \\
\hline No & 2,160 & 180 & 85.7 & 95.7 & 0.00002 \\
\hline Yes & 360 & 8 & 14.3 & 4.3 & \\
\hline \multicolumn{6}{|l|}{ Injury Location } \\
\hline Home & 66 & 1 & 2.4 & 0.5 & 0.052 \\
\hline Farm & 7 & 0 & 0.3 & 0.0 & $0.073^{\wedge}$ \\
\hline Mine & 13 & 0 & 0.5 & 0.0 & \\
\hline Industrial & 330 & 17 & 12.1 & 7.7 & \\
\hline Recreation & 51 & 1 & 1.9 & 0.5 & \\
\hline Street & 583 & 62 & 21.3 & 28.2 & \\
\hline Public Building & 237 & 21 & 8.7 & 9.5 & \\
\hline
\end{tabular}




\section{Cureus}

\begin{tabular}{|c|c|c|c|c|c|}
\hline Residential & 2 & 0 & 0.1 & 0.0 & \\
\hline Other & 1,301 & 110 & 47.5 & 50.0 & \\
\hline Unknown & 147 & 8 & 5.4 & 3.6 & \\
\hline \multicolumn{6}{|l|}{ Alcohol Involvement } \\
\hline No (by test) & 807 & 55 & 29.0 & 24.7 & 0.42 \\
\hline Yes, above legal limit & 672 & 56 & 24.2 & 25.1 & \\
\hline Yes, trace & 159 & 17 & 5.7 & 7.6 & \\
\hline Unknown & 1,142 & 95 & 41.1 & 42.6 & \\
\hline \multicolumn{6}{|l|}{ Drug Involvement } \\
\hline No (by test) & 492 & 34 & 17.7 & 15.2 & 0.024 \\
\hline Yes & 576 & 32 & 20.7 & 14.3 & \\
\hline Unknown & 1,712 & 157 & 61.6 & 70.4 & \\
\hline \multicolumn{6}{|l|}{ Payor } \\
\hline Government & 1,179 & 74 & 42.4 & 33.2 & $<10^{-6}$ \\
\hline Blue Cross/Blue Shield & 117 & 5 & 4.2 & 2.2 & \\
\hline Private/Commercial & 450 & 24 & 16.2 & 10.8 & \\
\hline Self & 565 & 81 & 20.3 & 36.3 & \\
\hline Other & 181 & 5 & 6.5 & 2.2 & \\
\hline Unknown & 288 & 34 & 10.4 & 15.2 & \\
\hline
\end{tabular}

\section{TABLE 4: Demographics by Survival}

$\wedge$ only for those injuries occurring at industrial properties, street, public buildings, or other

ICU: Intensive Care Unit; ISS: Injury Severity Score; LOS: length of stay; SD: standard deviation; US: United States

\section{Discussion}

There is little literature studying non-motor vehicle railway injuries in the US; the available studies are narrow in scope with small sizes $[5,8,10]$. The previous non-motor vehicle associated railway injury studies $[4,6,9-10]$ demonstrate a similar age: 39 years [6], 37 years [9], 31 years [4], and 21 - 40 years [10] compared to the 38.5 years in this study. There is also a male predominance in railway-associated injuries; we found that $80.6 \%$ of the injuries were in males, similar to $87 \%$ [4] and 59\% [6] in other studies. Males appeared to have sustained more severe injuries as indicated by their increased ISS, LOS, and ICU days, despite a younger average age. The average hospital LOS in our study of 12 days is consistent with 11 days in Sweden [6].

This is the first study to examine racial, geographical, and billing differences within railway injuries. The racial distribution seen in this study resembles that of the general US population [11]. Although certain racial groups were more commonly injured in different regions, this likely represents the racial distribution within the US. Railway injuries were most common in the South and least in the Northeast, even though the Northeast has three of the top five busiest Amtrak stations accounting for $80 \%$ of the Amtrak passenger traffic $(20,034,851$ of $25,139,294$ riders). The likely explanation for this is that the South has more miles of freight railroad compared to the Northeast, as the US is known to have much greater railway freight traffic compared to passenger traffic. In 2016, European railways carried 2.209 billion tons of freight, representing $6 \%$ of the worldwide rail freight; the worldwide percentage of freight carried for America was $29 \%, 36 \%$ for Asia, and 27\% for Russia. By contrast, the Worldwide Railway Organisation reported that 7.342 billion passengers were carried on trains in Europe in 2016 [1], with the worldwide percentage of passenger travel being $16 \%$ for Europe, $78 \%$ for Asia/Oceania, and $1 \%$ for America. This poses the question as to whether most of these injuries in this study occurred on or around freight railways as compared to passenger trains. If that is so, then the $71.4 \%$ of the patients that were designated as pedestrians or passengers were likely pedestrians and not passengers on the train. They were also likely trespassers [12], although such data is not given in the NTDB.

Work-related injuries were more frequent in men and less severe than non-work-related injuries (ISS $11.2 \mathrm{vs}$ 17.3), explaining the lower mortality rate (2.0\% vs $6.6 \%)$. The discrepancy in ISS could also be related to a lower threshold of workers seeking medical evaluation and presenting with less severe injuries compared to those with non-work-related injuries. Work-related injuries also rarely involved alcohol and were more commonly the result of a fall when compared to non-work injuries (32.8\% vs $17.9 \%)$. This increase in falls compared to non-work injuries could be related to employment needs/requirements, but the exact etiology is unclear. Interestingly, both work-related and non-work-related injuries had similar percentages for collision with stock ( $9.0 \%$ and $10.1 \%$, respectively), and hit by stock ( $24.4 \%$ and $28.7 \%$, respectively). This suggests that increased exposure and awareness of railways and their dangers do not necessarily decrease the incidence of stock-related injuries. 
those due to a fall, and the more severe injuries were due to those patients that were hit/collided with the stock or collided with an object. Those sustaining a fall were the least likely to be injured on the street and had the highest proportion of being injured in a public building and industrial locations. Similarly, they were the oldest of the various groups when grouped by injury mechanism. This likely means that those injured in public buildings were riders on the train, and those injured in industrial locations employees with workrelated injuries (Table 2). Conversely, those who were hit by stock more commonly sustained their injuries in "other" locations, which likely means the railroad itself. This may indicate that further passive governmental agencies (whether local, statewide, or national) create ways in which it is more difficult for non-sanctioned people to actually be on the railroad, such as more barriers to impede trespassers, etc. Active legislation will likely not work; passive measures are much better for injury prevention.

The role of alcohol in railway injuries is variable in the literature. Hedelin et al. [6] noted that $21 \%$ of nonfatal and $60 \%$ of fatal injuries involved alcohol above the legal limit, while our findings noted an approximately equal percentage of both non-fatal and fatal injuries ( $24.2 \%$ and $25.1 \%$, respectively). When recalculating the data of Hedelin et al. [6] using only the number of patients above the legal alcohol limit, the result is $23.9 \%$, the same as our result of $24.2 \%$. We noted that $29.1 \%$ of patients had some evidence of alcohol in their system compared to the $70 \%[4]$ and $80 \%[5,8]$ in other studies. In India, alcohol was detected in only $2.8 \%$ of 88 railway-related death victims examined post-mortem [10]. All of the studies mentioned above have sample sizes lower than 250 , which may explain these differences. The true percentage of patients under the influence of alcohol in train-related injuries is likely in the range corroborated by both our study and that of Hedelin et al. [6].

Previously published railway injury mortality rates are $17 \%$ [13], 14\% [4], and 10.4\% [6], compared to our $6.4 \%$. One explanation for these differences is that this study included all injury mechanisms that were classified as involving a railway and its premises, except those involving motor vehicles. Therefore, this study was not limited to only train/pedestrian events as seen in two of the studies [6, 13]. Higher mortality rates would be expected in those studies, as they would involve more severe injury mechanisms (such as hit by rolling stock which resulted in a $36.8 \%$ mortality rate in this study) while excluding less severe ones. Shapiro et al. [4] included motor vehicles, making it difficult to appropriately compare mortality rates with other studies excluding those injuries. All of the studies above, as well as in the present study, use hospital records to identify the patient population, which, unfortunately, neglects those who died on the scene and never presented to a hospital $[4,6,13]$. Thus, it is likely that the true railway injury mortality rate is higher than reported in all of these studies. The exact magnitude of this difference is difficult to know.

\section{Limitations}

The first limitation of this study is that the data entered into the NTDB is only as accurate as those entering the data. A recent study noted that there is interhospital variability in data coding and scoring with an overall $64 \%$ accuracy [14]. The accuracy, however, was good for standard demographic variables (96\%) and less accurate for physiologic variables, such as pre-hospital vital signs (36\%). Thus, the data for this study is likely very accurate as this is primarily a demographic study. Second, details of the ICD-9E codes regarding the mechanism of injury is not given, e.g., where exactly was the patient when hit by the rolling stock, etc. The passenger/pedestrian groups are not further broken down in the ICD-9 coding, so we could not study these subgroups. Another limitation is that not all patients have data entered for every variable; this was especially true for associated alcohol and drug use. This study is biased towards the more severe injuries, as all these patients were seen at trauma centers. It is very likely the more minor injuries, such as simple fractures, were seen and cared for at non-trauma center hospitals. The magnitude of this is difficult to know.

\section{Conclusions}

In this large study of the demographics of non-motor vehicle collision railway injuries treated at trauma centers, the average age was $38.5 \pm 17.1$ years with a male predominance of $80.6 \%$. The injuries were least common in the Northeast and most common in the Southern US. The racial distribution of the injured patients mirrored that of the US population. Alcohol involvement was present in $29.1 \%$ of patients, lower than in previous US studies. The overall mortality rate was $6.4 \%$, also lower than previously reported. These findings can be used by all involved in the care of such patients, as well as those in injury prevention.

\section{Additional Information \\ Disclosures}

Human subjects: Consent was obtained by all participants in this study. Indiana University Institutional Review Board issued approval Exempt. Under 45 CFR 46.101(b) and the SOPs, as applicable, the study is accepted as Exempt (4) Category 4: Secondary Use of Pre-Existing Data (Data must exist at the time the research is submitted for review.) Research involving the collection or study of existing data, documents, records, pathological specimens or diagnostic specimens if (i) these sources are publicly available, or (ii) the information is recorded by the investigator in such a manner that subjects cannot be identified, directly or through identifiers linked to the subjects. Animal subjects: All authors have confirmed that this study did not involve animal subjects or tissue. Conflicts of interest: In compliance with the ICMJE uniform disclosure form, all authors declare the following: Payment/services info: All authors have declared that no financial support was received from any organization for the submitted work. Financial relationships: All authors have declared that they have no financial relationships at present or within the previous three years with any organizations that might have an interest in the submitted work. Other relationships: All authors have declared that there are no other relationships or activities that could appear to have influenced the submitted work.

\section{Acknowledgements}

This research was supported, in part, by the Trauma Program, Riley Children's Hospital; the Garceau Professorship Endowment, Indiana University School of Medicine, Department of Orthopaedic Surgery; and the Rapp Pediatric Orthopaedic Research Endowment, Riley Children’s Foundation, Indianapolis, Indiana. 


\section{Cureus}

\section{References}

1. Railway Statistics 2016 Synopsis. (2017). Accessed: August 20, 2018: http://uic.org/IMG/pdf/synopsis_2016.pdf.

2. Bureau of Transportation Statistics: U.S. Passenger Miles 2016. (2019). Accessed: September 4, 2018 http://www.bts.gov/content/us-passenger-miles.

3. Federal Railroad Administration. Ten year accident/incident overview. Office of Public Safety . (2019). Accessed: August 20, 2018:

http://safetydata.fra.dot.gov/officeofsafety/publicsite/Query/TenYearAccidentIncidentOverview.aspx.

4. Shapiro MJ, Luchtefeld WB, Durham RM, Mazuski JE: Traumatic train injuries. Am J Emerg Med. 1994, 12:9293. 10.1016/0735-6757(94)90210-0

5. Moore TJ, Wilson JR, Hartman M: Train versus pedestrian accidents. South Med J. 1991, 84:1097-98 10.1097/00007611-199109000-00009

6. Hedelin A, Björnstig U, Brismar B: Trams--a risk factor for pedestrians . Accid Anal Prev. 1996, 28:733-38. 10.1016/S0001-4575(96)00048-6

7. Sousa S, Santos L, Dinis-Oliveira RJ, Magalhães T, Santo A: Pedestrian fatalities resulting from train-person collisions. Traffic Inj Prev. 2015, 16:208-12. 10.1080/15389588.2014.914181

8. Cina SJ, Koelpin JL, Nichols CA, Conradi SE: A decade of train-pedestrian fatalities: the Charleston experience. J Forensic Sci. 1994, 39:668-73. 10.1520/JFS13644]

9. Maclean AA, O'Neill AM, Pachter HL, Miglietta MA: Devastating consequences of subway accidents: traumatic amputations. Am Surg. 2006, 72:74-76.

10. Mohanty MK, Panigrahi MK, Mohanty S, Patnaik KK: Death due to traumatic railway injury. Med Sci Law. 2007, 47:156-60. 10.1258/rsmmsl.47.2.156

11. Quick Facts: United States. (2018). Accessed: September 4, 2018: http://www.census.gov/quickfacts/fact/table/US/PST045217.

12. Zhang M, Khattak AJ, Liu J, Clarke D: A comparative study of rail-pedestrian trespassing crash injury severity between highway-rail grade crossings and non-crossings. Accid Anal Prev. 2018, 117:427-38. 10.1016/j.aap.2018.02.001

13. Agalar F, Cakmakci M, Kunt MM: Train-pedestrian accidents. Eur I Emerg Med. 2000, 7:131-33.

14. Arabian SS, Marcus M, Captain K, et al.: Variability in interhospital trauma data coding and scoring: A challenge to the accuracy of aggregated trauma registries. J Trauma Acute Care Surg. 2015, 79:359-63. 10.1097/TA.0000000000000788 